\title{
Optical Surface Vortices and Their Use in Nanoscale Manipulation
}

\author{
M. Babiker ${ }^{a}$, V. E. Lembessis ${ }^{b}$ and D. L. Andrews ${ }^{c}$ \\ ${ }^{a}$ Department of Physics, University of York, Heslington, York YO10 5DD, England, UK \\ ${ }^{b}$ New York College of Athens, Athens, GR 105 58, Greece \\ ${ }^{c}$ School of Chemical Sciences, University of East Anglia, Norwich NR 4 7TJ, England, UK
}

\begin{abstract}
Following a brief overview of the physics underlying the interaction of twisted light with atoms at near-resonance frequencies, the essential ingredients of the interaction of atoms with surface optical vortices are described. It is shown that surface optical vortices can offer an unprecedented potential for the nanoscale manipulation of absorbed atoms congregating at regions of extremum light intensity on the surface.
\end{abstract}

Keywords: Surface Optical Vortices; Nanoscale; Optical Manipulation; Atoms at Surfaces. PACS: 42.50Tx; 42.50.Wk; 78.68+m; 37.10.De; 37.10.Vz

\section{INTRODUCTION}

With a combination of simple optical elements and a suitable laser, it is now possible to produce beams of light possessing unusual twisting properties [1-4]. In particular, the family of optical modes known as Laguerre-Gaussian beams, any desired member of which can now be readily created in the laboratory, is distinguished by an optical torque that is determined by the beam structure and phase properties. The discovery that these so-called 'twisted beams', also known as 'optical vortices', can produce turning effects on individual atoms, ions and molecules, and even larger particles, is arguably one of the most significant advances in optics in recent years. The twisting property is directly related to the orbital angular momentum carried by the light beams, which is in addition to any spin angular momentum associated with their wave polarisation. Since the discovery [1], this previously scarcely explored feature of laser light has generated much interest, not just from a fundamental point of view, but also with a view to its possible applications, especially the micro- and nanomanipulation of matter. The best known such application of twisted light to date has earned it the soubriquet 'optical spanner' [4], signifying a device capable of rotating small particles. Optical spanners are based on similar principles to optical tweezers [5] in which conventional laser light is capable of influencing the translation of small particles using the radiation pressure force. The rotations generated in optical spanners are due to the angular momentum property of the light beam. The essential physics underlying atomic manipulation by twisted light is based on the principles of cooling and trapping of neutral atoms and ions by ordinary laser beams. Theoretical work [6] has already established the existence of a light-induced torque acting on the 
centre of mass of an atom possessing a transition at near-resonance with the frequency of a Laguerre-Gaussian light beam carrying an orbital angular momentum $\hbar l$. The saturation form of this torque is $T \sim \hbar \Gamma l$, where $\Gamma$ is the width of the upper atomic state. The effects of this twisting effect on the motion of atoms and ions have been explored in a number of studies [7-10] leading to predictions of a localization of atoms in high intensity or dark regions of the light beam, suggesting a variety of possibilities for atom manipulation and trapping at the micro and nano-scales.

Recent experimental work on the optical interference between different LaguerreGaussian light beams has revealed a rich variety of intensity distributions. Two copropagating beams of equal and opposite values of $l$, but with slightly different frequencies produce a so-called 'optical Ferris wheel', in which the pattern rotates at frequency that depends on $l$ and the frequency separation of the two beams [11].

\section{SURFACE VORTICES}

The already established properties and behavior of optical vortices suggest that interesting effects might arise in an interference situation that involves twisted light suffering internal reflection at the surface between an optically dense medium and a vacuum. To our knowledge, this simple and easily realizable experimental scenario has not been investigated, nor has there been an exploration of its possible implications for surface atom manipulation using Laguerre-Gaussian light. Evanescent light due to conventional beams has, however, been successfully used in atomic mirrors [12] its use for manipulation at surfaces being considered for small objects [13-15], rather than atoms and molecules at near-resonance . For Laguerre-Gaussian beams, we show here that the evanescent light emerging in the vacuum region decays exponentially with distance normal to the surfaces. Significantly, its in-plane field distribution possesses the angular momentum properties of the incident beam. The emanating fields also include a plane surface wave with a wave-vector equal to the inplane component of the axial wave-vector of the incident beam. The overall field distributions form a surface optical vortex, endowed with an orbital angular momentum. A system of well defined incident beams, both of which are counterpropagating or both are co-propagating, can, in principle, be made to generate a corresponding set of evanescent surface optical vortices. There is, therefore, considerable scope for a variety of interference effects, possibilities for surface atom manipulation, and new surface optical tools akin to optical spanners and tweezers.

To begin, we specify the electric field of a Laguerre-Gaussian beam characterized by the Laguerre polynomial integer parameters $l$ and $p$; the beam is assumed to be traveling along the z-axis in a medium of a constant refractive index $n$ (the frequency dependence of the latter left implicit). Let $\omega$ be the frequency of the light and $k=n \omega / c$ the axial wavevector. For light plane-polarized along the $y$ axis the field vector can be written in cylindrical coordinates as;

$$
\boldsymbol{E}_{k l p}^{I}(r, \phi, z)=\hat{\boldsymbol{y}} F_{k l p}(r, z) e^{i(k z-\omega t)} e^{i l \phi}
$$

where $F_{k p}$ is an envelope function of $r$ and $z$. For the amplitude we have; 


$$
F_{k p}(r, z)=\frac{\varepsilon_{k 00}}{\left(1+z^{2} / z_{R}^{2}\right)^{1 / 2}}\left(\frac{r \sqrt{2}}{w(z)}\right)^{|l|} L_{p}^{\mid l}\left(\frac{2 r^{2}}{w^{2}(z)}\right) e^{-r^{2} / w(z)} e^{i \Theta_{G R}(z)}
$$

Here $\varepsilon_{k 00}$ is the amplitude for the corresponding plane wave of wave-vector $k$; and $w(z)$ is the beam waist at axial coordinate $z$ such that $w^{2}(z)=2\left(z^{2}+z_{R}{ }^{2}\right) / k z_{R}$ where $z_{R}$ is the Rayleigh range. The last phase factor in Eq. 2 exhibits the Guoy phase as well as the change in beam curvature with axial position. We also have;

$$
\Theta_{G R}(z)=\frac{k r^{2} z}{2\left(z^{2}+z_{R}^{2}\right)}+(2 p+|l|+1) \tan ^{-1}\left(\frac{z}{z_{R}}\right) .
$$

Note that the $z=0$ plane corresponds to the minimum beam waist $w(z)=w_{0}$, and that on this plane the Guoy and curvature term in Eq. 3 both vanish.

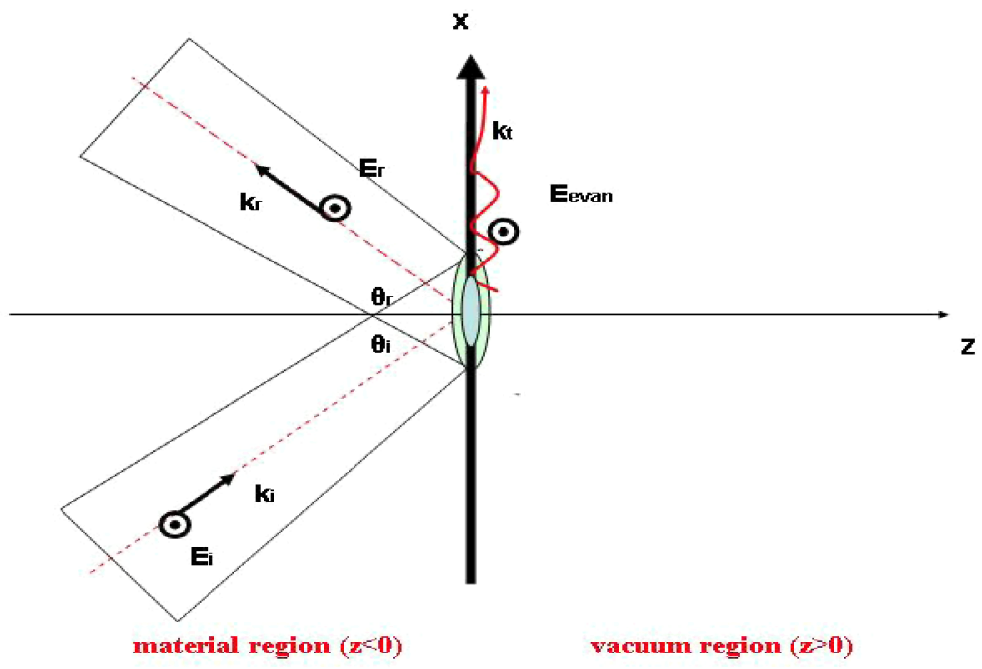

FIGURE 1: Laguerre-Gaussian light internally reflected at an angle $\vartheta$ greater than the critical angle (schematic). The incident beam is arranged such that at $\vartheta=0$ the beam waist coincides with the surface at $\mathrm{z}=0$. The evanescent light possesses angular momentum properties, but is confined near the surface, exponentially decaying in the direction normal to the surface.

Such a light field can be arranged, as shown in FIGURE 1, to strike the internal planar surface of a medium in which it is propagating, the surface assumed to be in contact with a vacuum. If the interface with the vacuum occupies the plane $z=0$ and the angle of incidence, $\vartheta$, exceeds the total internal reflection angle, an evanescent 
mode is created in the vacuum. The main requirement for the formation of this wave is the applicability of the standard phase-matching boundary condition, along with a requirement for the electric field vector component tangential to the surface to be continuous across the boundary. To be able to define the evanescent electric field we must first obtain expressions that are appropriate for a beam incident at an angle $\vartheta$. This is achievable by a simple rotational transformation of the expressions given in Eqs. (1) to (3).

The fields of a Laguerre-Gaussian beam propagating in a general direction can be constructed from the formalism outlined above by the application of two coordinate transformations, the first transformation rotating the beam as a rigid body about the $y$ axis by angle $\alpha$ and the second one rotates the resultant beam in the $z$ plane about the $x$ axis by an angle $\beta$. The compound transformation amounts to the following relations;

$$
\begin{aligned}
& x \rightarrow x^{\prime}=x \cos \alpha+z \sin \alpha \\
& y \rightarrow y^{\prime}=-x \sin \alpha \sin \beta+y \cos \beta+z \cos \alpha \sin \beta \\
& z \rightarrow z^{\prime}=-x \sin \alpha \cos \beta-y \sin \beta+z \cos \alpha \cos \beta
\end{aligned}
$$

By a suitable choice of the angles $\alpha$ and $\beta$ we can determine the field distributions of the incident $(\alpha=\vartheta ; \beta=0)$ and the internally reflected light $(\alpha=\pi-\vartheta ; \beta=0)$, as exhibited in FIGURE 1. The continuity of the electric field vector tangential to the surface, along with the exponential decay with the coordinate $z$ together determines the form of the evanescent filed in the vacuum region. We have;

$$
\boldsymbol{E}_{k l p}^{\text {evan }}(x, y, z)=2 \boldsymbol{E}_{k l p}^{I}(x \rightarrow x \cos \vartheta ; y ; z \rightarrow-x \sin \vartheta) e^{-z k_{0} \sqrt{n^{2} \sin ^{2} \vartheta-1}} e^{-i k_{0} n x \sin \vartheta}
$$

The explicit form of the evanescent electric field that displays the angular momentum properties, as well as the mode characteristics is as follows;

$$
\begin{aligned}
& \boldsymbol{E}_{k l p}^{\text {evan }}(x, y, z)=\hat{\boldsymbol{y}} \frac{\varepsilon_{k 00}}{\left(1+x^{2} \sin ^{2} \vartheta / z_{R}^{2}\right)^{1 / 2}}\left(\frac{\sqrt{2\left(x^{2} \cos ^{2} \vartheta+y^{2}\right)}}{w_{0}^{2}\left(1+x^{2} \sin ^{2} \vartheta / z_{R}^{2}\right)^{1 / 2}}\right)^{|l|} \\
& \times \exp \left(\frac{-\left(x^{2} \cos ^{2} \vartheta+y^{2}\right)}{w_{0}^{2}\left(1+x^{2} \sin ^{2} \vartheta / z_{R}^{2}\right)^{1 / 2}}\right) L_{p}^{l l}\left(\frac{x^{2} \cos ^{2} \vartheta+y^{2}}{w_{0}^{2}\left(1+x^{2} \sin ^{2} \vartheta / z_{R}^{2}\right)^{1 / 2}}\right) \\
& \times \exp \left(-z k_{0} \sqrt{n^{2} \sin ^{2} \vartheta-1}-i k_{0} n x \sin \vartheta\right) \exp \left[i l \tan ^{-1}(y / x \cos \vartheta)\right]
\end{aligned}
$$

Note that the above expression for the evanescent light bears a vorticity nature in that it is characterized by the azimuthal phase dependence $\exp \left[i l \tan ^{-1}(y / x \cos \vartheta)\right]$ of the incident and internally reflected light. The important point to bear in mind in this context is that the field distribution associated with this vortex is concentrated on the surface, rather than axially, as in the normal Laguerre-Gaussian light in an unbounded 
space.

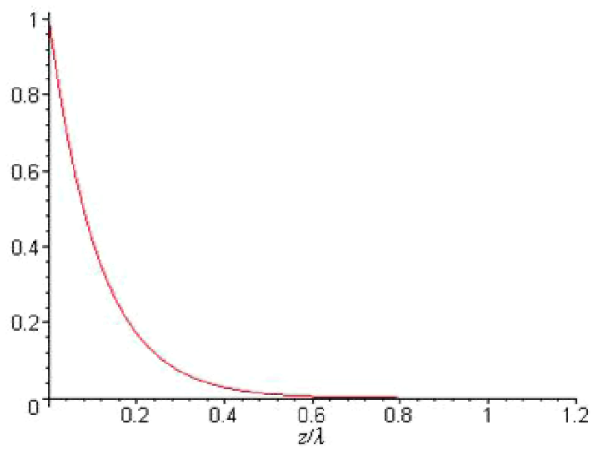

FIGURE 2: Intensity variations of the evanescent surface optical vortex (arbitrary units) with distance normal to the surface (in units of wavelength $\lambda$ ). This variation is independent of the values of $l$ and $p$.

The typical exponential decay of the evanescent light is shown in FIGURE 2. It is seen that the length scale of the exponential decay along $z$ spans a small fraction of the wavelength. In consequence, the evanescent light plays an unimportant role in the trapping normal to the surface - since adsorbed atoms are subject to the much stronger attractive van der Waal's potential.

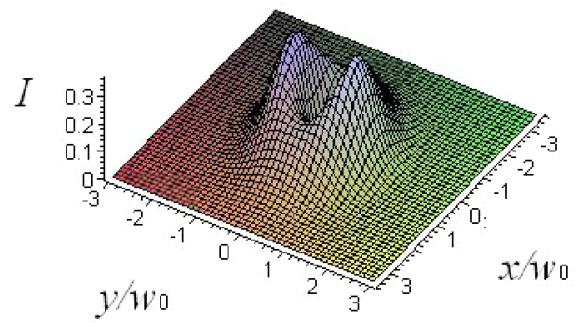

FIGURE 3: Intensity distribution (arbitrary units) on the surface $z=0$ for the surface vortex arising from internally reflecting a Laguerre-Gaussian light beam for which $l=1$ and $p=0$. Distances are measured in units of the beam waist $w_{0}$. The light has a wavelength $\lambda=589.16 \mathrm{~nm}$ and beam waist is taken as $w_{0}=35 \lambda$, corresponding to a long Rayleigh range $z_{R} \approx 2.27 \times 10^{-3} \mathrm{~m}$. The refractive index is assumed to be $n=\sqrt{2}$ and the angle of internal reflection is taken as $\vartheta=\pi / 3$, which is greater than the critical angle.

The intensity distribution corresponding to the evanescent light created by an incident Laguerre-Gaussian beam for which $l=1 ; p=0$ is shown in FIGURE 3, plotted in the $x-y$ plane at $z=0$. It is seen that the evanescent light possesses welldefined intensity maxima and minima that can be used to trap adsorbed atoms that have transition frequencies appropriately detuned from the frequency $\omega$ of the light. Note that the profile of the intensity distribution is no longer circular, but elliptical, because the light strikes the surface at the angle of incidence $\vartheta$, with an ellipticity increasing with increasing $\vartheta$. 


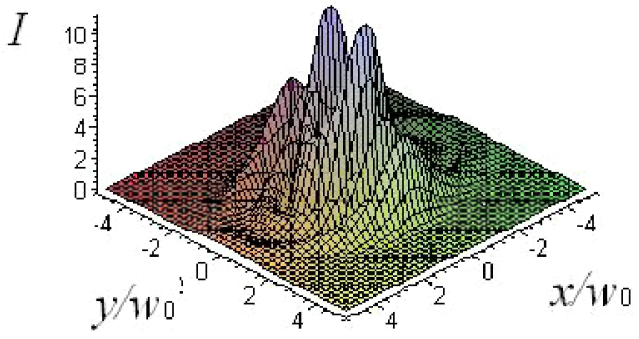

FIGURE 4: As in Figure 3, but here the Laguerre-Gaussian beam is such that $l=3$ and $p=2$.

The case $l=3 ; p=2$, with the same parameters as in earlier figures, is shown in FIGURE 4. This case presents a more complex field distribution for the corresponding surface optical vortex. Clearly the field distributions for any values of the parameters $l$ and $p$ can be obtained in a similar manner, suggesting that it is in principle possible to create a surface vortex of any order, confirming that the surface optical vortex phenomenon is quite general.

Consider now the case of co-propagating incident beams of opposite helicity creating an interference of two surface vortices, in a manner similar to that discussed recently for beams in an unbounded space, leading to the phenomenon of an optical Ferris wheel [11]. It is easy to see that the total electric field generated in the vacuum region contains the following factor;

$$
\cos \left[l \tan ^{-1}(y / x \cos \vartheta)\right]
$$

The appearance of the cosine term in Eq. 7 indicates an interference of the two evanescent light beams in the azimuthal direction.

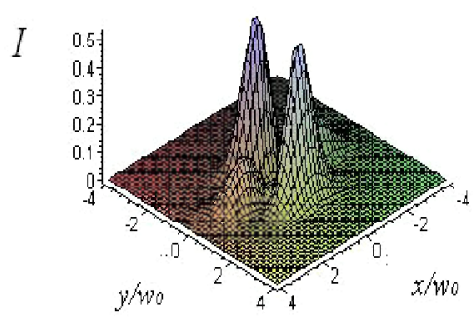

(a)

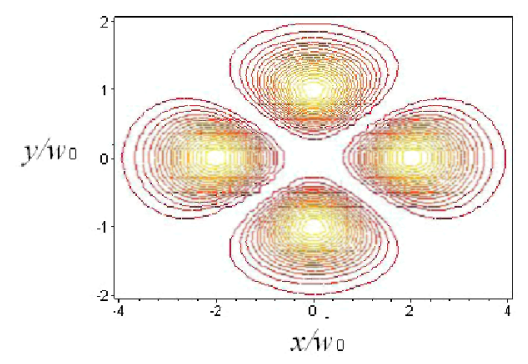

(b)

FIGURE 5: (a) Intensity distribution (arbitrary units) on the surface at $z=0$ arising from two copropagating internally reflected Laguerre-Gaussian light beams for which $l_{1}=2 ; p_{1}=0$ and $l_{2}=-2 ; p_{2}=0$ (b) the corresponding contour plot. The variables and parameters are as in FIGURES 
3 and 4 . If the beams are slightly different in frequencies such that $\omega_{1}-\omega_{2}=\delta$, the intensity distribution pattern is predicted to rotate at a frequency $\delta /\left(2\left|l_{1}-l_{2}\right|\right)$.

FIGURE 5 shows the field distributions for the case $l_{1}=2 ; p_{1}=0$ and $l_{2}=-2 ; p_{2}=0$. If the frequencies of the beams differ slightly, the pattern will rotate at a frequency $\delta /\left(2\left|l_{1}-l_{2}\right|\right)$ with $\delta$ the frequency difference, in a similar manner to that illustrated in the case of optical Ferris wheel in unbounded pace [11].

\section{CONCLUSIONS}

In conclusion, we have outlined the essential physics underlying the new phenomenon of surface optical vortices, associated particularly with LaguerreGaussian light. We have shown that the intensity distribution for a typical surface optical vortex has a limited spatial extent in the direction normal to the surface in the vacuum region, extending significantly over a distance of the order of a fraction of a wavelength. However, the in-plane distributions mimic those of the incident internally reflected light and carry the signature of orbital angular momentum of the incident beam. Since the optical vortices of a Laguerre-Gaussian kind form a family of vortices of any desired order defined by the integers $l$ and $p$, the phenomenon is quite general.

In addition to the intrinsic importance of surface optical vortices as physical entities in their own right - especially the fact that they carry the property of orbital angular momentum - there are some applications that can be envisaged at this stage. We have briefly mentioned the manipulation of adsorbed atoms and molecules held on the surface by the strong van der Waal's force, but these are commonly able to migrate along the surface. There has recently been strong interest in the manipulation of such atoms on surfaces, but none of the methods suggested so far include near-resonance optical manipulation along the surface. Optical vortices have the potential for the manipulation of adsorbed atoms, forcing them to congregate in extremum regions of intensity on the surface. The same principle can be employed to create patterned surfaces by employing carefully designed sets of incident beams creating a network of evanescent light wells. Moreover, the same system can be used to manipulate larger objects on the surface as optical tweezers and optical spanners, by moving the spot at which the incident beam strikes the interface. Finally, it can be observed that surface optical vortices of other kinds can be generated from other optical beams carrying orbital angular momentum, and that enhancement effects of the evanescent light can be realized by introducing a metallic film, leading to an evanescent surface plasmon mode [16] in which the filed intensities can be at least an order of magnitude larger. Work on surface plasmon optical vortices and guided modes is now in progress and the results will be reported in due course. 


\section{ACKNOWLEDGMENTS}

VEL wishes to thank the ESF for financial support under the programme QUDEDIS Exchange Grant 1750, while this work was carried out.

\section{REFERENCES}

1. L. Allen, M. W. Beijesbergen, R. J. C. Spreeuw, and J. P. Woerdman, Phys. Rev. A 45, 8185 (1992).

2. L. Allen, M. J. Padgett, and M. Babiker, Prog. Optics, vol. XXXIX, pp291-372 (1999).

3. L. Allen, S. M. Barnett, and M. J. Padgett, 'Optical Angular Momentum' (Institute of Physics Publishing, 2003).

4. D. L. Andrews, Ed. 'Structured Light and its Applications, an Introduction to Phasestructured Beams and Nanoscale Optical Forces ' (Academic, Burlington MA, 2008).

5. Special journal issue on optical tweezers, J. Mod. Opt. 50, $1501 \mathrm{ff}$ (2003).

6. M. Babiker, W. L. Power and L. Allen, Phys. Rev. Lett. 73, 1239 (1994).

7. M. Babiker, C. R. Bennett, D. L. Andrews, and L. C. D'avila Romero, Phys. Rev. Lett. 89, 143601 (2002).

8. D.L. Andrews, A.C. Carter, M. Babiker, and M. Al-Amri, Proc. SPIE 6131, 101-110 (2006).

9. A.R. Carter, M. Babiker, M. Al-Amri, and D. L. Andrews, Phys. Rev. A 72, 043407 (2005).

10. A. R. Carter, M. Babiker, M. Al-Amri, and D. L. Andrews, Phys. Rev. A 73, 021401 (2006).

11. S. Franke-Amold, J. Leach, M. J. Padgett, V. E. Lembessis, D. Ellinas, A. J. Wright, J. M. Girkin, P. Ohberg and A. S. Arnold, Opt. Express 15, 8619 (2007).

12. See C. R. Bennett, J. B. Kirk and M. Babiker, Phys. Rev. A 63, 033405 (2001), and references therein

13. V. Garce's-Chavez, K. Dholakia and G. C. Spalding, Appl. Phys. Lett. 86, 031106 (2005).

14. P. J. Reece, V. Garce's-Chavez and K. Dholakia, Appl. Phys. Lett. 88, 221116 (2006).

15. L. C. Thomson, G. Whyte, M. Mazilu and J. Courtial, J. Opt. Soc. Am. B 25, 849 (2008).

16. W. L. Barnes, A. Dereux and T. W. Ebbesen, Nature 424, 824 (2003). 